\title{
'|||||||||||||||||||||||||||||||||||||||||||||||||||||||||||||||||||.
}

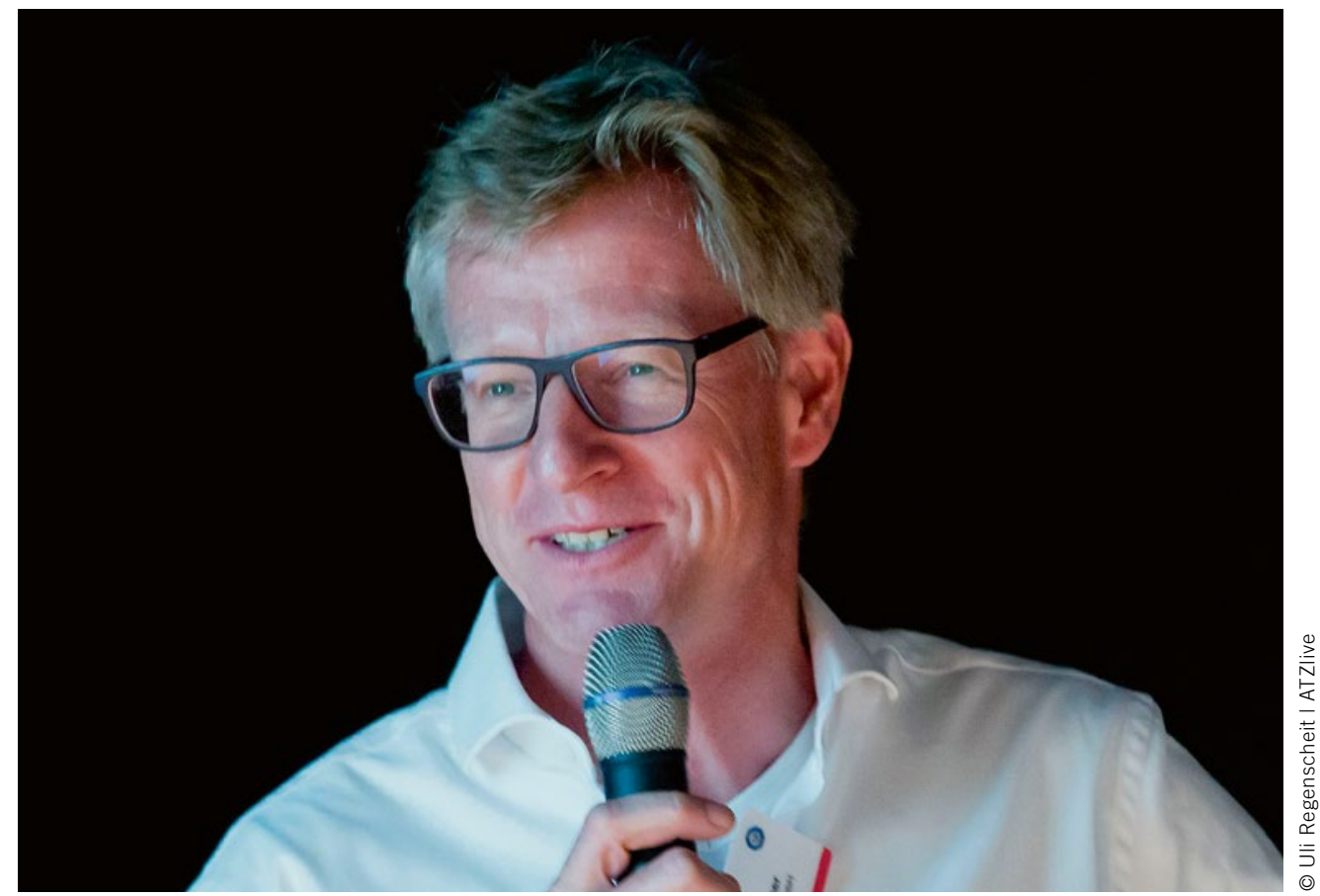

Dipl.-Ing. Frank Esser

Supervisor

Advanced Driving Attribute

Methods \& Tools,

Chassis and Vehicle Architecture

\& Technology,

Ford Research \& Advanced

Engineering,

ATZ-Beirat

\section{Wenige Prototypen - gute Fahrdynamik}

Der Ansatz ist keinem von uns neu, wird die Fahrdynamikentwicklung schon lange dazu aufgefordert, die Anzahl der physikalischen Prototypen zu reduzieren. Auch vervielfacht sich der Applikationsaufwand durch die neu aufkommenden elektrifizierten Antriebsversionen, speziell was die Auslegung der aktiven Chassiselemente und Assistenzfunktionen betrifft.

Wie kann nun ein neuer Weg in der Chassis- und Fahrdynamikentwicklung aussehen? Kann das Zusammenspiel zwischen ADAS und klassischer Fahrdynamik funktionieren? In allen Häusern wird intensiv daran gearbeitet, vom extrem teuren Vollfahrzeugprototypen in der frühen Bauphase wegzukommen und sowohl die funktionale Verifizierung als auch das Basistuning ins Labor oder die CAE-Welt zu verschieben. Folgendes muss dafür erfüllt sein: Die Komponenten- und Systemmodelle müssen ausreichend validiert, die Schnittstellen klar definiert und die funktionalen Ziele exakt beschrieben sein.

Dies erfordert essentiell eine frühe und intensive Zusammenarbeit zwischen dem OEM und seinen Zulieferern. Dazu zählen der frühzeitige Austausch über die Werkzeugketten, die Erwartungshaltung an die Qualität der Modelle, die notwendige Validierung und gemeinsame Definition der Schnittstellen.

Und wie kann das die Optimierung im Detail durch uns Experten unterstützen? Aus meiner Sicht liegt die Basis hier in der gemeinsamen Arbeit an den Modellen. Dies sollte beim Modell der Komponente beginnen, sich über das System fortsetzen und in der Vollfahrzeugsimulation enden. Dabei ist es wichtig, sich gemeinsam über Folgendes klar zu werden: Reicht mir ein Modell, das die Eigenschaften der Komponente wiedergibt, oder benötige ich ein physikalisch basiertes Modell, das mir eine Optimierung im Design der Komponente ermöglicht?

Diese und weitere Fragen lassen sich nur von OEM und Zulieferer gemeinsam klären. Der OEM braucht dazu hohe Kompetenz im Bereich der virtuellen Entwicklung, aber auch der dazu gehörenden Modellparametrisierung und nötiger physikalischer Tests. Genauso wichtig ist jedoch ein in diesem Bereich kompetenter Zulieferer. Ein wichtiger Schritt, um die virtuelle Entwicklung auf OEM-, aber auch Zuliefererseite effizient zu realisieren, ist die Schaffung entsprechender Strukturen und eine definierte Integration in den Entwicklungsprozess. So wie für den Prototypenbau klare Phasen definiert sind, so muss es auch für den virtuellen Prozess sein.

Mit der heutigen Komplexität der Varianten ist es kaum noch zu schaffen, alle Varianten physisch zu testen und erst recht auf Robustheit zu optimieren. Die Zeit und die Teile dafür sind nicht vorhanden. Hier setzt das gut validierte Modell an, mit dem die Vollfahrzeugeigenschaften abgetestet und optimiert werden können. Dazu muss das Modell gut verstanden sein, man muss wissen, für welchen Bereich es validiert ist, wo man sich auf das Modell verlassen kann. Großes Potenzial sehen wir darin, die finale Abstimmung auf Prototypen aus der Vorserienproduktion zu verlagern. 